\title{
Controls of the hydraulic safety-efficiency trade-off
}

Charlotte Grossiord $^{1,2}$, Danielle E. M. Ulrich ${ }^{3}$, Alberto Vilagrosa ${ }^{1,4}$

${ }^{1}$ Swiss Federal Research Institute WSL, Zürcherstrasse 111, 8903 Birmensdorf,

Switzerland

${ }^{2}$ École Polytechnique Fédérale de Lausanne EPFL, School of Architecture, Civil and

Environmental Engineering ENAC, 1015 Lausanne, Switzerland

${ }^{3}$ Department of Ecology, Montana State University, Bozeman, MT, 59717 USA

${ }^{4}$ CEAM Foundation, Joint Research Unit University of

Alicante-CEAM, Dept Ecology, Univ of Alicante, PO Box 99, 03080 Alicante, Spain.

\section{Total word count: 1744}

Number of Figures: 1

This document is the accepted manuscript version of the following article:

Grossiord, C., Ulrich, D. E. M., \& Vilagrosa, A. (2020). Controls of the hydraulic safetyefficiency trade-off. Tree Physiology. https://doi.org/10.1093/treephys/tpaa013 
A major trade-off when acquiring $\mathrm{CO}_{2}$ from the atmosphere during photosynthesis is the loss of water from plant leaves. Transporting as much water as quickly as possible with little carbon investment (often defined as hydraulic efficiency and usually measured as xylem-specific hydraulic conductivity) can lead to higher potential transpiration and photosynthesis per unit xylem cross-section area. However, critical dysfunctions such as embolisms and cavitation by water stress can form inside the xylem and constrain the efficiency of water transport (Tyree \& Sperry, 1989; Vilagrosa et al., 2003). Plant xylem tissues therefore require a certain degree of safety (usually measured as the xylem water potential at which a meaningful percentage of maximum efficiency is lost; Gleason et al., 2016).

As tissue-level hydraulic safety and efficiency are both determined by structural characteristics of the xylem network, plants are thought to confront a fundamental tradeoff between xylem-level efficiency and safety (i.e. negative correlation, Fig. 1) to obtain and transport water to their leaves as efficiently as they can, while minimizing the risk of drought-induced cavitation. For example, efficient plants with wider conduits tend to have more interconduit pits and to possess a large membrane-pore through which embolism and cavitation may occur (Wheeler et al., 2005). Thus, a specific suite of xylem traits that confers high efficiency could also reduce its safety. Although a trade-off between efficiency and safety may take place at the scale of the xylem (e.g. smaller pit membrane pores increase safety but reduce efficiency), this tissue-level trade-off may weaken at larger scales like whole membranes or conduits (e.g. through changes in conduit features such as a greater number of interconduit pits to compensate for the reduction in pit membrane pores), and even the whole plant (Meinzer et al., 2010). However, evidence of a safety-efficiency trade-off are not widespread in the literature (e.g. Tyree et al., 1994; Maherali et al., 2004), and even at the scale of the xylem global studies have revealed that plants often exhibit both low efficiency and safety (e.g. Gleason et al., 2016).

How efficient $v s$. how safe a plant is, and thus its position along a hypothetical safety-efficiency trade-off (Fig. 1) could depend on genetic adaptation of plants to their climate of origin: plants originating from drier climates may tend to have "safer" 
hydraulic traits compared to plants from more mesic environments that may tend to have “efficient” hydraulic traits (e.g. Nardini \& Luglio, 2014; Schumann et al., 2019). Genetic adaptation (i.e. all genetic changes of a species in response to selective pressure exerted by its local environment) is considered a major mechanism that enables plants to moderate the negative impacts associated with varying environmental conditions, including extreme events like droughts. However, while plants with a short life cycle may adapt quickly genetically, slow-growing long-lived organisms like trees may not be able to adapt fast enough to a rapidly changing climate (e.g. McLachlan et al., 2005). In this context, phenotypic plasticity (i.e. reversible dynamic shifts in phenotype in response to environmental pressure) may be more advantageous for trees to persist with more frequent and extreme droughts predicted for the future. Moreover, plasticity in hydraulic traits could help species by providing them with greater safety during dry periods and greater efficiency during more favorable conditions (Fig. 1). The magnitude of phenotypic plasticity and genetic adaptation, and the impacts on plant physiology during extreme climate events have been analyzed in many studies comparing species in different ecosystems or biomes (Sultan, 2000; Pigliucci, 2001; DeWitt \& Scheiner, 2004), but thus far, few have distinguished the genetic vs. plastic controls on the hydraulic safety-efficiency trade-off. In this issue of Tree Physiology, Pritzkow et al. shed new insight on the genetic and plastic drivers of the hydraulic safety-efficiency trade-off.

By combining a field and a nursery study, Pritzkow et al., examined the intraspecific variation in canopy and xylem hydraulic traits of Eucalyptus obliqua, an evergreen species that naturally spans a large precipitation gradient in southeastern Australia, and thus is likely to show a safety-efficiency trade-off between populations from mesic $v s$. dry environments. In their field study, the authors quantified seasonal variability in a large array of aboveground hydraulic traits related to efficiency (e.g. maximum hydraulic conductivity) and safety (e.g. P50/P88 corresponding to the water potential at $50 \%$ and $88 \%$ loss of hydraulic conductivity). For the nursery study, the authors collected seeds from individuals at each field site and grew individuals in a glasshouse. As initially hypothesized by the authors, their findings demonstrate a strong genetic control of several hydraulic traits related to hydraulic safety (e.g. leaf size, leaf 
vulnerability to embolism), which overall suggests that populations from mesic environments may be at higher risk of hydraulic failure during drought (i.e. low safety but high efficiency) compared to populations from drier environments. However, evidence for phenotypic plasticity in physiological and anatomical hydraulic traits related to both hydraulic safety and efficiency was found for all trees along the gradient (e.g. turgor loss point, Huber value), and thus may allow E. obliqua populations, including the most vulnerable populations in mesic environments, to acquire greater safety during extreme events. As suggested by the authors, underlying processes driving these changes could involve leaf- and branch-level short-term adjustments such as an extension of the range of water potentials under which the cells remain functional or a reduction of leaf area per unit sapwood area through leaf shedding.

The strength of the approach used by Pritzkow et al. relies on the combination of field and controlled conditions to identify phenotypically plastic traits in the field and also to detect traits under genetic control. The field study allowed the authors to determine the amplitude of phenotypic plasticity in response to drought stress in diverse populations of E. obliqua along a precipitation gradient. Pritzkow et al.'s nursery study, like common gardens or provenance trials, is specifically designed to separate the effects of genetic control and phenotypic plasticity on functional trait expression. As shown in previous works (e.g. Mencuccini \& Comstock, 1999; Sparks \& Black, 1999; Choat et al., 2007; Aranda et al., 2014, Kerr et al., 2015), the findings of Pritzkow et al. confirm that a large array of hydraulic traits are genetically controlled. Pritzkow et al. also confirm that populations originating from wetter environments may be at higher risk of hydraulic failure during drought because when grown in the nursery, they expressed functional traits that make them more efficient than safe. Furthermore, the amplitudes of phenotypic plasticity in single traits along the precipitation gradient were minor, suggesting that subtle changes in a suite of traits (e.g. leaf area, $\mathrm{P}_{50} / \mathrm{P}_{88}$ ) can be effective in adapting to specific habitats (e.g. Rosas et al., 2019). This result highlights the value of evaluating multiple traits in field and common garden studies.

With the projected exacerbation of drought with global warming, mesic populations may thus be more likely to experience drought-induced mortality and to be subjected to reduced primary productivity if they are not able to shift their functional 
traits towards greater safety faster than the projected onset of warmer and drier conditions. This begs several questions about populations from more mesic climates: how will mesic populations fare under the predicted warmer, drier conditions, and are hydraulic traits from mesic populations more plastic relative to dry populations to compensate for the greater risk of being highly efficient? Although these important questions come to mind while reading this work, they remain unanswered given this study's experimental design. Indeed, population-specific differences in the amplitude of plasticity cannot be inferred from the work of Pritzkow et al., as trees in natural field sites were not exposed to the same soil moisture stress during the measurements. The literature does not provide a clear answer to this question either. Some studies have found that species originating from wetter environments can exhibit greater plasticity than drier populations (e.g. Corcuera et al., 2011) while others have observed higher plasticity at the very dry limit of the species distribution range (e.g. González-Muñoz et al., 2018), but a lack of clear information is the general rule. The discrepancy in the patterns of responses, including the results of Pritzkow et al., may relate to the strong focus on only aboveground traits, which could be leading to an over-simplification of plant plastic responses to drought stress so far.

Although measuring belowground traits is more challenging than aboveground ones, it is obvious that to provide strong relevance, traits should be determined in all levels, from the roots to the canopy (Fortunel et al., 2012). In particular, dynamic physiological belowground traits related to safety and efficiency such as deep-water uptake (i.e. safety) and high root hydraulic conductivity (i.e. efficiency) deserve increased attention in the context of drought impacts (Grossiord et al., 2017), compared to the more common morphological traits that are usually studied (e.g. leaf size and area, xylem anatomy, Huber value). While plasticity in aboveground physiological functions has been the focus of numerous studies (Grams et al., 2007), the degree of plasticity occurring belowground (e.g. seasonal variability in water sources or root hydraulic conductivity) could reveal new strategies that would alter our understanding of plant responses to drought. Further, legacy effects (e.g. embolism that carries over between droughts) and specific components of plasticity, such as ecological memory (i.e. the capability of past experience of an individual to influence future plastic responses, Ogle 
et al., 2015), must be investigated to fully unravel the significance of phenotypic plasticity in the context of plant survival under drought. Such stress memory that plants maintain after an initial stress could lead to greater plasticity in subsequent extreme events (Walter et al., 2011), while legacy effects might make trees more vulnerable to future droughts events because of reduced hydraulic capacity (Wu et al., 2017). However, as most studies focus on short-term plastic responses, it is still unclear on which time scales such plant ecological memory acts with the few studies available indicating adjustments varying over periods of days (Resco de Dios et al., 2016) to a growing season (Walter et al., 2011).

Overall, the results of Pritzkow et al. provide an intriguing new perspective on the safety-efficiency trade-off, and the role of short-term plastic responses on this conceptual framework. However, untangling the full significance of the safety-efficiency trade-off to provide modelers with reliable predictive frameworks on forest responses to changing climate will require future work to investigate all components of the genetic and plastic controls of hydraulic safety $v s$. efficiency, including looking at what takes place belowground, over multiple years, and more than one generation. 


\section{Acknowledgments and Funding}

CG was supported by the Swiss National Science Foundation SNF (5231.00639.001.01). AV was supported by WSL visiting fellowship and IMAGINA project (Prometeo program 2019/110- Generalitat Valenciana). CEAM is supported by Generalitat Valenciana.

\section{Conflict of interest}

None declared.

\section{Contributions}

C.G., D.E.M.U and A.V. developed the conceptual framework and wrote the manuscript. 


\section{References}

Aranda, I., Cano, F.J., Gascó, A., Cochard, H., Nardini, A., Mancha, J.A., López, R. and Sánchez-Gómez, D., 2014. Variation in photosynthetic performance and hydraulic architecture across European beech (Fagus sylvatica L.) populations supports the case for local adaptation to water stress. Tree physiology, 35(1), pp.34-46.

Choat, B., Sack, L. and Holbrook, N.M., 2007. Diversity of hydraulic traits in nine Cordia species growing in tropical forests with contrasting precipitation. New Phytologist, 175(4), pp.686-698.

Corcuera, L., Cochard, H., Gil-Pelegrin, E. and Notivol, E., 2011. Phenotypic plasticity in mesic populations of Pinus pinaster improves resistance to xylem embolism (P 50) under severe drought. Trees, 25(6), pp.1033-1042.

DeWitt, T. J., \& Scheiner, S. M. (Eds.). 2004. Phenotypic plasticity: functional and conceptual approaches. Oxford University Press.

Fortunel, C., Fine, P.V. and Baraloto, C., 2012. Leaf, stem and root tissue strategies across 758 Neotropical tree species. Functional Ecology, 26(5), pp.1153-1161.

González-Muñoz, N., Sterck, F., Torres-Ruiz, J.M., Petit, G., Cochard, H., von Arx, G., Lintunen, A., Caldeira, M.C., Capdeville, G., Copini, P. and Gebauer, R., 2018.

Quantifying in situ phenotypic variability in the hydraulic properties of four tree species across their distribution range in Europe. PLoS One, 13(5), p.e0196075.

Gleason, S.M., Westoby, M., Jansen, S., Choat, B., Hacke, U.G., Pratt, R.B., Bhaskar, R., Brodribb, T.J., Bucci, S.J., Cao, K.F. and Cochard, H., 2016. Weak tradeoff between xylem safety and xylem-specific hydraulic efficiency across the world's woody plant species. New Phytologist, 209(1), pp.123-136.

Grams, T. E., \& Andersen, C. P., 2007. Competition for resources in trees: physiological versus morphological plasticity. In Progress in botany (pp. 356-381). Springer, Berlin, Heidelberg.

Grossiord, C., Sevanto, S., Dawson, T.E., Adams, H.D., Collins, A.D., Dickman, L.T., Newman, B.D., Stockton, E.A. and McDowell, N.G., 2017. Warming combined with 
more extreme precipitation regimes modifies the water sources used by trees. New Phytologist, 213(2), pp.584-596.

Kerr, K.L., Meinzer, F.C., McCulloh, K.A., Woodruff, D.R., Marias, D.E., Expression of functional traits during seedling establishment in two populations of Pinus ponderosa from contrasting climates. Tree physiology 35(5), pp. 535-548.

Maherali, H., Pockman, W.T., Jackson, R.B., 2004. Adaptive variation in the vulnerability of woody plants to xylem cavitation. Ecology 85, pp. 2184-2199.

McLachlan, J. S., Clark, J. S., \& Manos, P. S. 2005. Molecular indicators of tree migration capacity under rapid climate change. Ecology, 86(8), pp. 2088-2098. Meinzer, F.C., McCulloh, K.A., Lachenbruch, B., Woodruff, D., Johnson, D.M. 2010. The blind men and the elephant: the impact of context and scale in evaluating conflicts between plant hydraulic safety and efficiency. Oecologia 164: pp. 287-296.

Mencuccini, M. and Comstock, J., 1999. Variability in hydraulic architecture and gas exchange of common bean (Phaseolus vulgaris) cultivars under well-watered conditions: interactions with leaf size. Functional Plant Biology, 26(2), pp.115-124.

Nardini, A. and Luglio, J., 2014. Leaf hydraulic capacity and drought vulnerability: possible trade-offs and correlations with climate across three major biomes. Functional Ecology, 28(4), pp.810-818.

Ogle, K., Barber, J.J., Barron Gafford, G.A., Bentley, L.P., Young, J.M., Huxman, T.E., Loik, M.E., Tissue, D.T., 2015. Quantifying ecological memory in plant and ecosystem processes. Ecology Letters 18, pp. 221-235.

Pigliucci, M. 2001. Phenotypic plasticity: beyond nature and nurture. JHU Press.

Rehfeldt, G.E., Ying, C.C., Spittlehouse, D.L., Hamilton, D.A., 1999. Genetic responses to climate change in Pinus contorta: niche breadth, climate change, and reforestation. Ecological Monographs 69, pp. 375- 407.

Resco de Dios, V., Loik, M.E., Smith, R., Aspinwall, M.J. and Tissue, D.T., 2016. Genetic variation in circadian regulation of nocturnal stomatal conductance enhances carbon assimilation and growth. Plant, Cell \& Environment, 39(1), pp.3-11. 
Rosas, T., Mencuccini, M., Barba, J., Cochard, H., Saura-Mas, S. and Martínez-Vilalta, J., 2019. Adjustments and coordination of hydraulic, leaf and stem traits along a water availability gradient. New Phytologist, 223(2), pp. 632-646.

Sparks, J.P. and Black, R.A., 1999. Regulation of water loss in populations of Populus trichocarpa: the role of stomatal control in preventing xylem cavitation. Tree physiology, 19(7), pp.453-459.

Sultan, S. E., 2000. Phenotypic plasticity for plant development, function and life history. Trends in plant science 5(12), pp. 537-542.

Schumann, K., Leuschner, C., \& Schuldt, B., 2019. Xylem hydraulic safety and efficiency in relation to leaf and wood traits in three temperate Acer species differing in habitat preferences. Trees, pp. 1-16.

Tyree, M.T. and Sperry, J.S., 1989. Vulnerability of xylem to cavitation and embolism. Annual review of plant biology, 40(1), pp.19-36.

Tyree, M.T., Davis, S.D., Cochard, H. 1994. Biophysical perspectives of xylem evolution - is there a tradeoff of hydraulic efficiency for vulnerability to dysfunction. IAWA Journal 15, pp. 335-360.

Vilagrosa, A., Bellot, J., Vallejo, V.R. and Gil-Pelegrín, E., 2003. Cavitation, stomatal conductance, and leaf dieback in seedlings of two co-occurring Mediterranean shrubs during an intense drought. Journal of experimental botany, 54(390), pp.2015-2024.

Walter, J., Nagy, L., Hein, R., Rascher, U., Beierkuhnlein, C., Willner, E. and Jentsch, A., 2011. Do plants remember drought? Hints towards a drought-memory in grasses. Environmental and Experimental Botany, 71(1), pp.34-40.

Wheeler, J.K., Sperry, J.S., Hacke, U.G., Hoang, N. 2005. Inter-vessel pitting and cavitation in woody Rosaceae and other vesselled plants: a basis for a safety versus efficiency trade-off in xylem transport. Plant, Cell \& Environment 28: pp. 800-812. Wu, X., Liu, H., Li, X., Ciais, P., Babst, F., Guo, W., Zhang, C., Magliulo, V., Pavelka, M., Liu, S. and Huang, Y., 2018. Differentiating drought legacy effects on vegetation 
growth over the temperate Northern Hemisphere. Global change biology, 24(1), pp.504516. 


\section{Figure legend}

\section{Figure 1: Theoretical representation of the hydraulic efficiency-safety trade-off. A}

theoretical trade-off between hydraulic efficiency and safety is expected (highlighted in light orange, but see Gleason et al. 2016): populations more frequently exposed to warm and dry conditions (population B) tend to have safer xylem hydraulic traits (e.g. smaller xylem vessel diameters, lower xylem vulnerability to embolism) relative to populations from wetter and more moist environments (population A) that tend to be more efficient in their resource use (e.g. higher maximum hydraulic conductivity, higher turgor loss point). The position along the efficiency-safety trade-off should depend on genetic controls (i.e. adaptation, population A vs. B) but phenotypic plasticity (highlighted by dashed lines) allows populations to expand strategies, including gaining a safer one during drought (movement towards higher hydraulic safety) and a more efficient one during wetter periods. The magnitude of plasticity (i.e. difference between dotted lines and red circles) is unknown but highly efficient populations may require a greater trait plasticity and ability to adapt to persist with projected drought exacerbation as they are genetically at higher risk of drought-induced mortality (i.e. low safety).

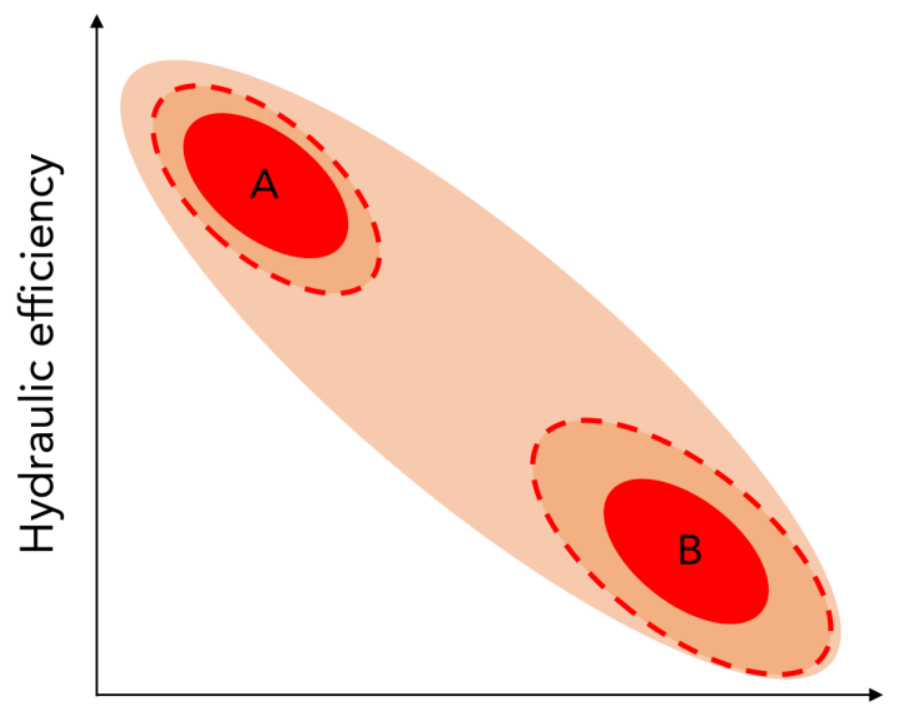

Hydraulic safety 